\title{
Wireless Luminaires
}

\begin{abstract}
The development in the field of lighting technology is focused on new technologies, light sources with higher efficiency and better lighttechnical parameters and the development of luminaires, especially in the field of design and small light sources. The efficiency of light sources is associated with converting of electricity into the light in the entire process chain, including the efficiency of power wires and ballast systems. It can be stated that the losses in the power supply line and wires path are less important in comparison with the actual process of internal energy conversion in light sources.

Since the beginning of use of electrical energy for the conversion to light the conductor and wires are used. The luminaires are fixed to one place and move with them is limited to a small place. The solution may be a wireless power and energy transfer. The result of this solution is the possibility of placing the luminaires anywhere in a defined space without wires and cables. The disadvantage is significantly lower efficiency of energy transfer and strong electromagnetic interference, which may exceed the limits of EMC and affect the operational of other electrical equipments. Questionable issue is the exposure of human body to RF fields unnecessarily. This paper deals with the search for potentially useful applications of wireless powering in lighting.
\end{abstract}

Keywords: Wireless; Wireless Luminaires; Wireless Energy Transfer; Magnetic Induction

\section{Introduction}

Wireless luminaires have place in only a few applications. An example can be exhibitions, discos, thematic lighting and special cases. Generally, all applications are low power at maximum input power of $10 \mathrm{~W}$. The efficiency of wireless transfer is dependent on frequency and transfer distance. The value of efficiency is in range from 10 to $80 \%$ and operating frequency in the order of hundreds $\mathrm{kHz}$ or $\mathrm{MHz}$.

Theoretically can be used three basic principles of energy transfer. The first one is the transmission by the magnetic field, which is based on magnetic induction $B$. The second method is based on transmission by electric high frequency field. And the last principle uses high frequency electromagnetic radiation from the powerful magnetrons.

Individual principles have advantages and disadvantages, which are described in the following sections. Some applications are implemented in the laboratory, including measured operating parameters.

\section{Wireless Magnetic Induction}

The transfer of energy by magnetic induction is one of the oldest and most commonly used current ways. All wireless chargers, computer mice and small applications use this method. The transmission principle is described in Figure 1. The source of high frequency supply current is the feedback high-frequency oscillator. The relative permeability of air is very low and the value is close to $\varepsilon r=1$. Operating frequency of oscillator must be at least of the order of tens of $\mathrm{kHz}$, because all magnetic circuit is created by air.
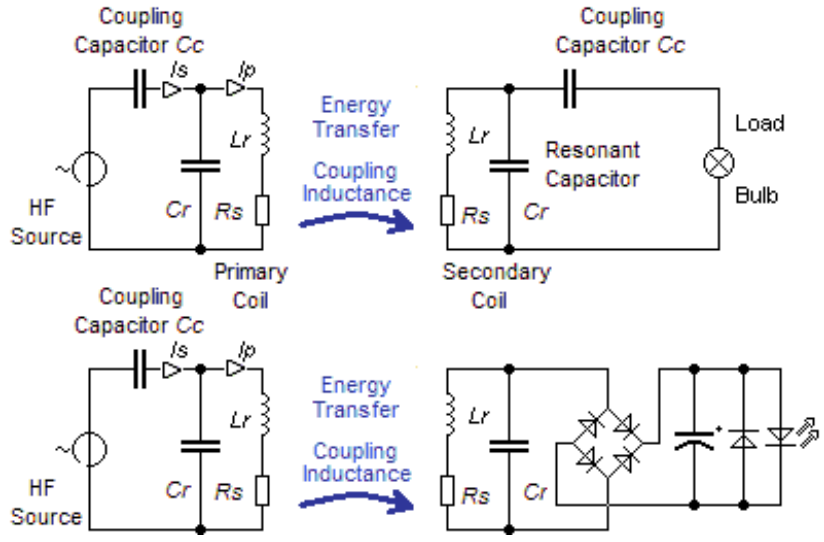

Fig.1. Schematic of energy transfer by magnetic induction
The resonant circuit on the transmitter side may be serial or parallel. However, transfer efficiency is dependent on the value of magnetic induction $B$, which is determined by a value of current passing trough the transmitting inductor. From this perspective, it is preferable a parallel resonant circuit. The current passing through the inductor $I p$ is $Q$ times larger than the powering current $I s$. The value $Q$ is called the quality of resonant circuit and its value is primary affected by the parasite conductance of resonant capacitor $G$ and series resistance of inductor $R$. Therefore, the parallel resonant circuits are created only by quality film capacitors and solid copper conductors. This ensures that the current in transmitting circuit will be in order of tens or hundreds of $A$. And a sufficiently strong magnetic filed will be created by this value of current for power transmission over long distance.

The receiving circuit is also tuned parallel resonant circuit. The capacitor acts as an impedance matching to the load. For example, the classical bulb can be connected directly to the resonant circuit, but LEDs must be connected via a rectifier and voltage limiter.

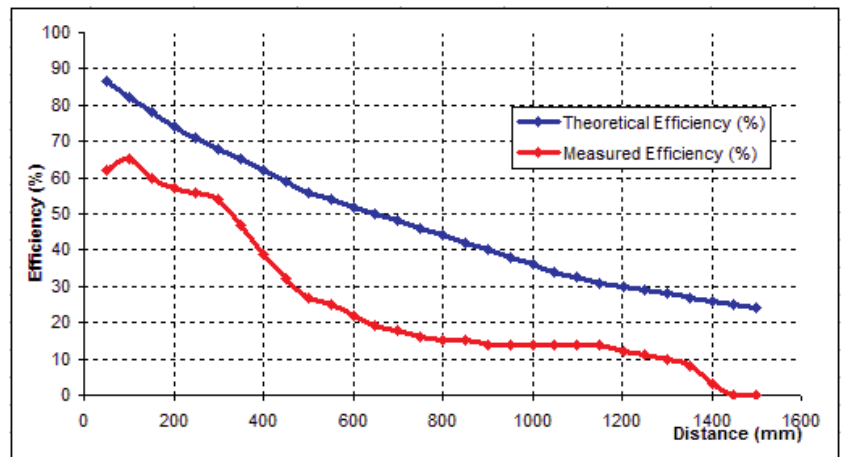

Fig.2. Theoretical and measured efficiency of transfer

The magnetic induction and the associated transmission efficiency depend on the transfer distance. The value of efficiency would be changed exponentially if the transmitter and receiver will a point source. But in fact the dependence is different when are taken physical dimensions of the resonant circuits. Theoretical and practical efficiency dependence on the distance of energy transfer is shown in Figure 2. The transmitting and receiving antenna with diameter of $240 \mathrm{~mm}$ is used for calculate. 
A significant electromagnetic interference is the main disadvantage in addition to the low efficiency of energy transfer. The operation of electric equipment nearby the high frequency transfers may be affected by high-energy radiation. The telecommunication transmission and especially the radio transmission in $\mathrm{AW}$ and $\mathrm{LW}$ are disturbed. The RF magnetic field has a negative accumulation character for human body. It is therefore necessary to ensure that nobody is needlessly permanently positioned near these facilities.

\section{One-wire Magnetic Induction}

The low power luminaires can be also powered from the high frequency current loop. Each luminaire is equipped with a ferrite transformer. Light source is connected to the winding of this transformer. The current loop is fed from high frequency power oscillator. Luminaire is movably connected via loop wire. Each luminaire can be powered via several different loops, which enables to create complex wiring. The principle is depicted in Figure 3 and the possibilities for complex wiring in Figure 4. An illustrative example of implementation photograph is shown in Figure 5. These applications are safe from the perspective of electric shock, nowhere does not occur dangerous contact voltage.

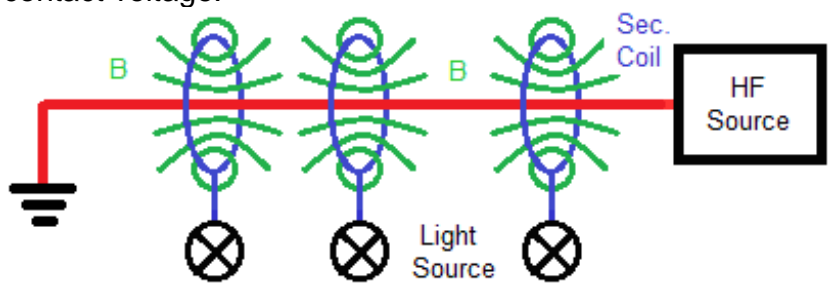

Fig.3. Principle of One-wire Magnetic Induction

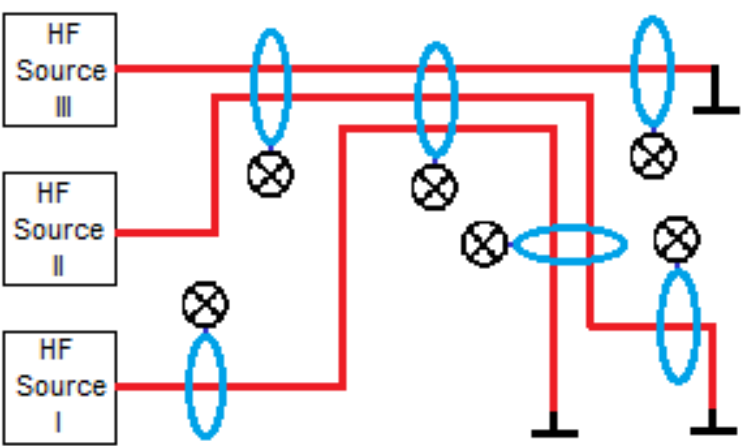

Fig.4. Principle of Complex Wiring

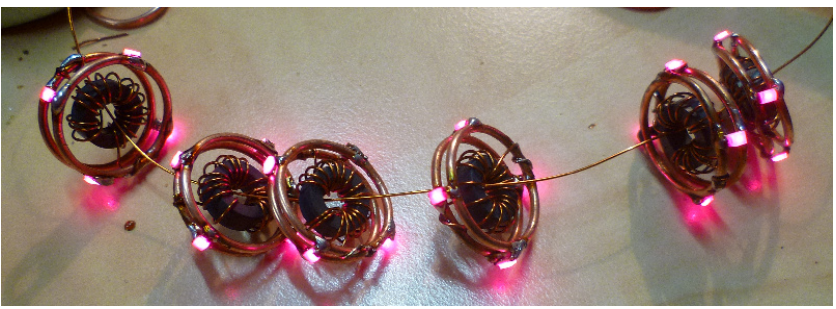

Fig.5. Photography of Implementation

\section{Electric High Frequency Field}

Electric field can be better formed and distributed in comparison with magnetic field. It can be also easily shielded. Sources of high frequency electric field are RF high voltage laboratory sources, such as Tesla coil. Electric field intensity $E p$ up to several $\mathrm{kV} / \mathrm{cm}$ is created by these sources. The electric field intensity $E p$ is decreased exponentially with distance. The places with different voltage potential are created. Electric current is passed through conductive objects, which are inserted between these two different voltage potential. Examples are discharge lamps in which the light or UV radiation are formed by direct passing of current. The energy transfer based on the electric field is shown in Figure 6. Image of energy transfer by Tesla coil is depicted in Figure 7.
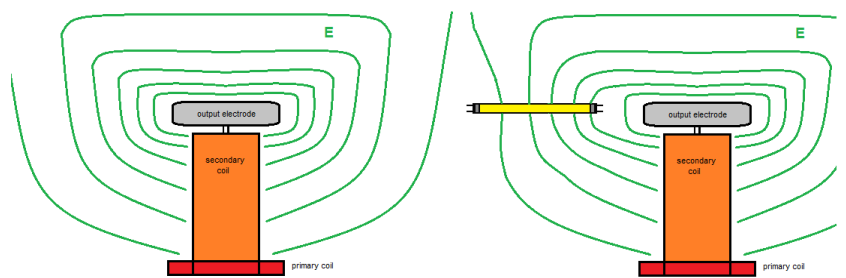

Fig.6. Distribution of Electric Field

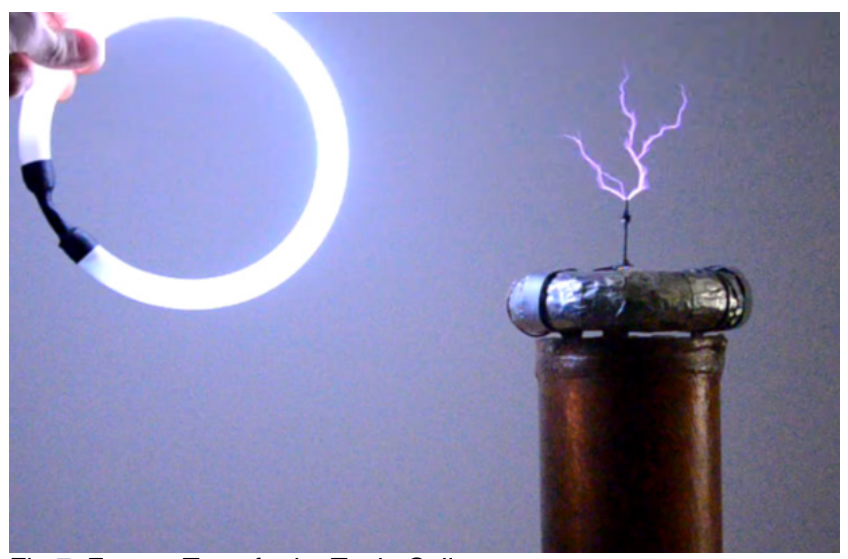

Fig.7. Energy Transfer by Tesla Coil

\section{Electromagnetic Radiation}

The last example of wireless energy transfer is based on high energy microwave radiation. Today a great variety of magnetrons and their relatively low prices allow to wirelessly supplied energy to light sources. Examples include sulphur and LIFI lamps. However, in the same way may be excited also other discharge lamps. Again there are problems of shielding hazardous radiation by shielding metal and covers. Therefore, this principle is described theoretically, no practice testing. The Figure 8 shows the arrangement schematic of this method.

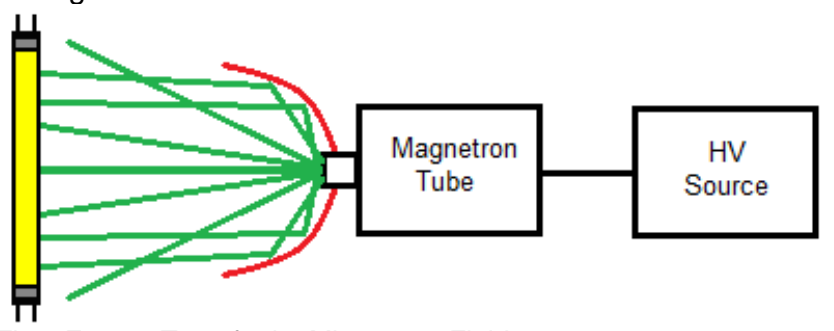

Fig.8 Energy Transfer by Microwave Field

\section{Implementation and Measured Data}

In our laboratory were designed, implemented and parameter measured 3 models of wireless power transmission.

The first model is self-resonant autotuned oscillator with operational frequency of approximately $1 \mathrm{MHz}$. Maximum power consumption of this model is $15 \mathrm{~W}$ at a supply voltage of $15 \mathrm{~V}$, but maximum input voltage is limited up to $30 \mathrm{~V}$. The primary resonant circuit is realized by a singleturn inductor with a diameter of $240 \mathrm{~mm}$. The inductor is made of $10 \mathrm{~mm}$ cooper tube. Capacity of resonant capacitor is $60 \mathrm{nF}$. And the measured inductor current is approximately $50 \mathrm{~A}$. The receiver circuit is made of same 
components. At the output of receiver circuit is connected rectifier, filtration and $1 \mathrm{~W}$ LED Osram Oslon.

With the distance the output power to the LED is decreased rapidly. At distance of $1 \mathrm{~m}$ output power is only $100 \mathrm{~mW}$ and at distance of $1.3 \mathrm{~m}$ the LED is turned off. The photography of this device is shown in Figure 9.
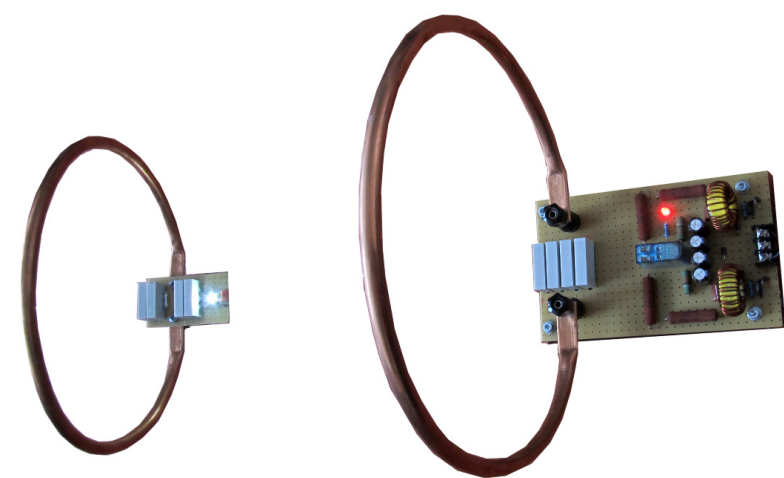

Fig.9 Photography of Energy Transfer by Magnetic Induction

The dependence of measured efficiency and value of magnetic induction on the distance from the transmitter is featured in Figure $10 \mathrm{~A}$ and the measured distribution of magnetic field of transmitter coil is depicted in Figure $10 \mathrm{~B}$.

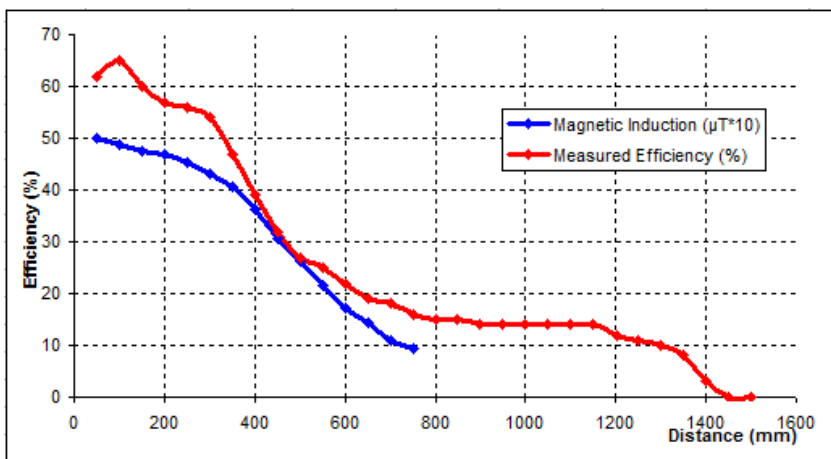

Fig.10 A Measured Efficiency of Energy Transfer

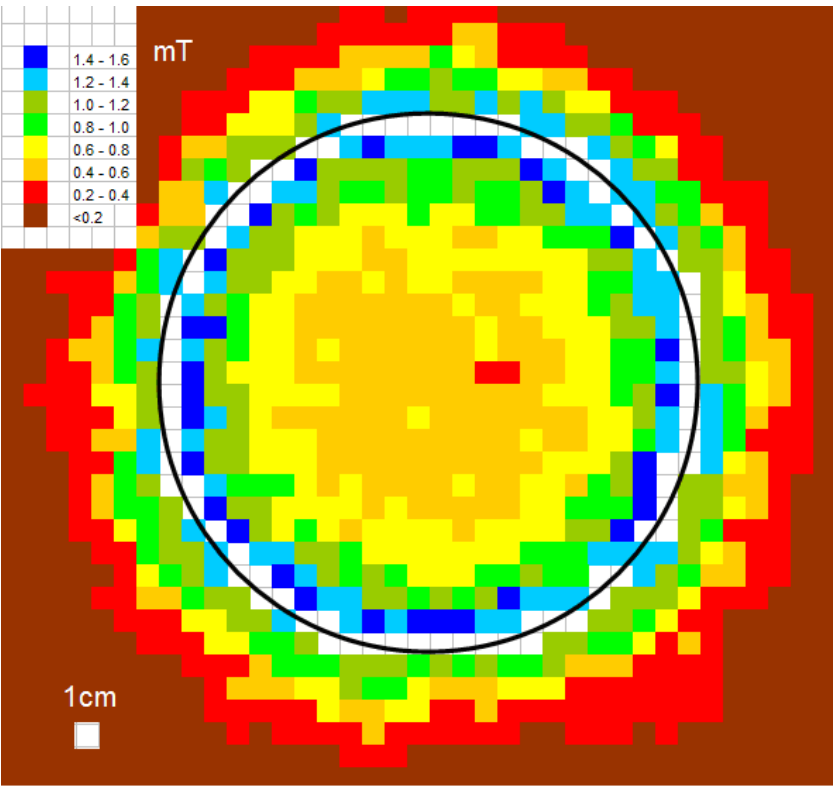

Fig.10 B Distribution of Magnetic Field of Transmitter Coil

Transfer efficiency was calculated from the input power of the whole oscillator and the output power of the LED.

The second model is based on the same principle. The main difference is in the lower operating frequency $(40 \mathrm{kHz})$ and large physical dimension of transmitter coil. The transmission distance and transfer efficiency is lower due to lower operating frequency, in comparison with the first model. The distribution of magnetic induction in the plate of transmitter coil is shown in Figure 11.

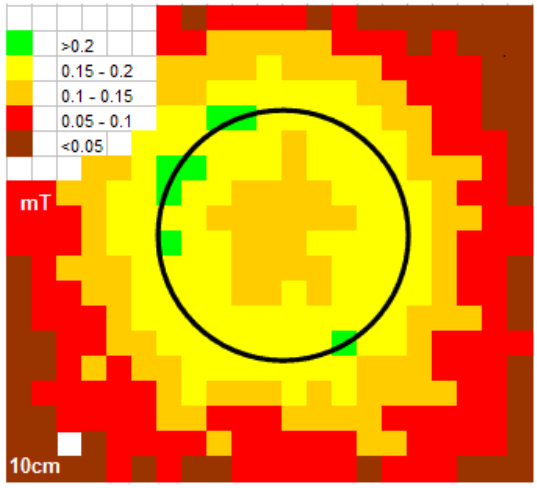

Fig.11 Distribution of Magnetic Field of Transmitter Coil II

The energy transfer by electric field is used in the last laboratory model. The transmitter is represented by the Tesla coil with operating frequency of $600 \mathrm{kHz}$ and input power of $100 \mathrm{~W}$. Mode of operating is continuous and the output voltage reaches the value of $20 \mathrm{kV}$ at output electrode with diameter of $120 \mathrm{~mm}$. High intensity of electric field $E$ enables the formation of different voltage potentials. It is necessary to achieve the value of intensity of electric filed of at least $100 \mathrm{~V} / \mathrm{cm}$ for proper operation of embedded fluorescent lamp tubes. Measured electric field distribution near the transmitting electrodes is depicted in Figure 12.

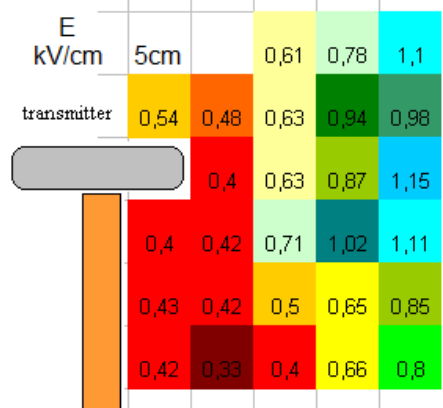

Fig.12 Measured Intensity of Electric Field around Tesla Coil

\section{Conclusion}

From the measured values obtained in laboratory is apparent that wireless luminaires can be used for lowpower applications. Low efficiency of energy transfer is the main disadvantage. The strong interference of radio waves is also necessary to consider.

\section{Acknowledgments}

This research work has been carried out in the Centre for Research and Utilization of Renewable Energy (CVVOZE). Authors gratefully acknowledge financial support from the Ministry of Education, Youth and Sports of the Czech Republic under NPU I programme (project No. LO1210) and BUT specific research programme (project No. FEKT-S-14-2520).

\section{REFERENCES}

[1] Cheon S., Circuit - Model - Based Analysis of a Wireless Energy-Transfer System via coupled Magnetic Resonances, IEEE TRANSACTIONS ON INDUSTRIAL ELECTRONICS, VOL. 58, NO. 7, JULY 2011 
[2] NICULAE D., IORDACHE M., DUMITRIU L., Magnetic Coupling Analysis in Wireless Transfer Energy, University POLITEHNICA Bucharest

[3] J.A.Ricano, H. Rodriguez, H. Vasquez "Experiment About Wireless Energy Transfer", 1-st International congress on instrumentation and applied sciences, Cancun, Mexico, october 2010

[4] N. Tesla "Apparatus for transmitting electrical energy", U.S patent number1119732, issued in December 1914;

[5] Liu L., Zhang R., Wireless Information Transfer with Opportunistic Energy Harvesting, IEEE TRANSACTIONS ON WIRELESS COMMUNICATIONS, VOL. 12, NO. 1, JANUARY 2013

[6] Zeng Y., Optimized Training Design for Wireless Energy Transfer, IEEE TRANSACTIONS ON COMMUNICATIONS, VOL. 63, NO. 2, FEBRUARY 2015
[7] Seungyoung A., Cho D., Future Wireless Power Transportation System, 2013 Asia-Pacific Microwave Conference Proceedings

[8] Kurs A., Wireless power transfer via strongly coupled magnetic resonances Science Express, Vol. 317. no. 5834, pp. 83 - 86, June 2007.

Authors: Ing. Michal Krbal, Ph.D., Ing. Jan Škoda, Ph.D., Ing. Jaroslav Štěpánek, doc. Ing. Petr Baxant, Ph.D., Vysoké učení technické v Brně - Brno University of Technology, Fakulta elektrotechniky a komunikačních technologií, Technická 12, Czech Republic, e-mail: krbal@feec.vutbr.cz 